\title{
Differential Transient MEG and fMRI Responses to Visual Stimulation Onset Rate
}

\author{
August S. Tuan, ${ }^{1,2,3}$ Rasmus M. Birn, ${ }^{3}$ Peter A. Bandettini, ${ }^{3}$ Geoffrey M. Boynton ${ }^{2,4}$ \\ ${ }^{1}$ Department of Neurosciences, University of California, San Diego, La Jolla, CA 92093 \\ 2 The Salk Institute for Biological Studies, La Jolla, CA 92037 \\ ${ }^{3}$ The Laboratory of Brain and Cognition, National Institute of Mental Health, Bethesda, MD 20892 \\ ${ }^{4}$ Department of Psychology, University of Washington, Seattle, WA 98195
}

Received 16 October 2007; revised 18 April 2008; accepted 24 April 2008

\begin{abstract}
While recent analysis of functional magnetic resonance imaging (fMRl) data utilize a generalized nonlinear convolution model (e.g., dynamic causal modeling), most conventional analyses of local responses utilize a linear convolution model (e.g., the general linear model). These models assume a linear relationship between the blood oxygenated level dependent (BOLD) signal and the underlying neuronal response. While previous studies have shown that this "neurovascular coupling" process is approximately linear, short stimulus durations are known to produce a larger fMRI response than expected from a linear system. This divergence from linearity between the stimulus time-course and BOLD signal could be caused by neuronal onset and offset transients, rather than a nonlinearity in the hemodynamics related to BOLD contrast. We tested this hypothesis by measuring MEG and fMRI responses to stimuli with ramped contrast onsets and offsets in place of abrupt transitions. MEG results show that the ramp successfully reduced the transient onset of neural activity. However, the nonlinearity in the fMRI response, while also reduced, remained. Predictions of fMRI responses from MEG signals show a weaker nonlinearity than observed in the actual fMRI data. These results suggest that the fMRI BOLD nonlinearity seen with short duration stimuli is not solely due to transient neuronal activity. (c) 2008 Wiley Periodicals, Inc. Int J Imaging Syst Technol, 18, 17-28, 2008; Published online in Wiley InterScience (www.interscience.wiley.com). DOI 10.1002/ima.20144
\end{abstract}

Key words: functional MRI; nonlinearity; MEG; transient onset; visual cortex

\section{INTRODUCTION}

Functional magnetic resonance imaging (fMRI) has grown as a powerful noninvasive tool for studying and detecting patterns of activation in the human brain. While there are many variants of fMRI, the most prevalent is blood oxygenated level dependent

Correspondence to: August S. Tuan; e-mail: august@salk.edu

Grant sponsor: National Institute of Mental Health Intramural Research Program.
(BOLD) fMRI. The BOLD signal is thought to reflect changes in deoxyhemoglobin content in local venous microvasculature (Belliveau et al., 1991; Bandettini et al., 1992; Kwong et al., 1992; Ogawa et al., 1992). These changes in deoxyhemoglobin concentration correlate with neuronal activity and are dependent on a complex interaction between cerebral metabolic rate of oxygen $\left(\mathrm{CMRO}_{2}\right)$, cerebral blood flow $(\mathrm{CBF})$, and cerebral blood volume (CBV) (for review, see Heeger and Ress, 2002).

A relationship is linear if it satisfies the properties of scaling and superposition-a weighted sum of input waveforms produces a weighted sum of individual output responses (Miller et al., 2001). Ideally, the transformation between neuronal activity and BOLD signal should be linear so that the fMRI response reflects the underlying neuronal response averaged over a small region of space and a short period of time. Although it is unlikely that the BOLD signal is truly a linear system due to the complexity of the neurovascular coupling process, even an approximation of linearity would greatly simplify the analysis and interpretation of the fMRI signal.

Nearly all fMRI data analysis methods assume a linear relationship between the BOLD signal and the underlying neuronal response. The assumption of linearity is supported by previous studies that show, to a first approximation, the fMRI response can be predicted by a linear convolution in time of the physical stimulus (Boynton et al., 1996; Dale and Buckner, 1997; Vazquez and Noll, 1998; Glover, 1999). If the transformation between stimulus attributes and the neuronal response is linear, then this means that the fMRI response to a long duration stimulus can be predicted by the fMRI response to a short duration stimulus and the fMRI response to a high contrast stimulus can be predicted by the fMRI response to a low contrast stimulus. However, brief stimuli (lasting less than 3 or $4 \mathrm{~s}$ ) have been shown to produce disproportionately large fMRI responses relative to longer duration stimuli in visual and auditory cortices (Boynton et al., 1996; Robson et al., 1998; Vazquez and Noll, 1998). For example, 250-ms stimulus can produce an fMRI 
response that is three to five times larger than predicted from the response to a longer stimulus, and this nonlinearity can vary within and across cortical areas (Birn et al., 2001).

It is unclear whether this is due to a nonlinear neuronal response, a nonlinear hemodynamic response, or to a combination of both. Recent studies have shown evidence of a nonlinearity between CBF and BOLD and in particular, hemodynamic refractoriness has been suggested to explain why shorter duration stimuli have disproportionately larger responses (Mechelli et al., 2001; Miller et al., 2001; Obata et al., 2004). There is supporting evidence of this from fMRI and optical imaging experiments that show how the hemodynamic response to a stimulus is strongly affected by previous stimuli (Dale and Buckner, 1997; Cannestra et al., 1998; Friston, 1998; Huettel and McCarthy, 2000, 2001; Inan et al., 2004).

An alternative hypothesis is that the BOLD nonlinearity in visual (Boynton et al., 1996) and auditory (Robson et al., 1998) cortex is caused by rapid neuronal adaptation, where a large burst of initial activity quickly decays to a weaker sustained response (Albrecht et al., 1984; Maddess et al., 1988; Bonds, 1991). Positron emission tomography experiments also show an increase then reduction in activity of word processing areas following $600 \mathrm{~ms}$ of exposure to displayed words (Price and Friston, 1997). Electrophysiological data has shown large transients in the neuronal response at the stimulus onset and offset (Albrecht et al., 1984; Muller et al., 1999, 2001). Since these transients presumably occur equally for stimuli of all durations, they should have a disproportionately larger influence on the average response for shorter stimulus durations than for longer stimulus durations. This could explain the larger relative BOLD signal to stimuli that last less then $4 \mathrm{~s}$. Neuroimaging studies suggest that neuronal onset transients may be the source of identifiable BOLD onset transients that may be used to characterize schizophrenia (Fox et al., 2005a,b).

In this study, we examined nonlinearities in the mapping between stimulus function and observed fMRI BOLD responses. These nonlinearities could occur in the mapping between the stimulus function (e.g., a boxcar function encoding the presence of a stimulus) and the neuronal response. Alternatively, nonlinearities may occur between the evoked neuronal response and the fMRI BOLD response. There may also be nonlinearities between both the stimulus function-to-neuronal mapping and the neuronal-to-hemodynamic mapping. We first measured a surrogate of neuronal activity using time-frequency analyses of magnetoencephalography (MEG). MEG has been utilized as a surrogate marker for neuronal activity in a number of studies investigating anatomical localization, cortical processing, and neuronal networks (Orrison et al., 1990; Hari and Forss, 1999; David et al., 2003; Momjian et al., 2003). The high temporal resolution of MEG allowed us to quantify nonlinear effects such as neuronal adaptation at the level of neuronal responses, in relation to nonlinear effects such as hemodynamic refractoriness in the mapping of neuronal activity to the observed BOLD response. At the neuronal level, these nonlinear effects often manifest as onset or offset transients and related adaptation phenomena. Briefly, we demonstrate that there is indeed a profound nonlinear component to neuronal responses that was not sufficient to account for the refractoriness of hemodynamic response, suggesting that there are both neuronal and hemodynamic contributions.

\section{MATERIALS AND METHODS}

A. Subjects. Thirteen subjects underwent a complete physical examination and provided informed consent (fMRI experiment, $N=$ 10; MEG experiment, $N=5$; two subjects participated in both experiments). All subjects had normal or corrected-to-normal visual acuity. All subjects were free of neurological or psychiatric illness and were compensated for participation in the study, and anatomical MR scans were screened by the NIH Clinical Center Department of Radiology in accordance with the National Institute of Mental Health Institutional Review Board guidelines.

\section{B. MEG Experiment.}

B.1. MEG Stimulus Presentation Apparatus. Stimuli for the MEG experiments were generated on an Apple PowerMac G3 laptop computer (Apple Computers, Cupertino, CA) using Matlab version 5.2 (MathWorks, Natick, MA) and the Psychophysics Toolbox (Brainard, 1997; Pelli, 1997). Stimuli for the MEG experiments were produced using a Sharp PG-210U projector (Camus, WA) fitted with a zoom lens, located outside of the scanner room, and passed the images through a guide tube. During MEG data acquisition, subjects were placed in a seated position and directly viewed the image on a back-projection screen located $74 \mathrm{~cm}$ in front of the subject. Subjects' heads were stabilized with an airbladder and chinstrap system.

B.2. MEG Stimulus. In both our MEG and fMRI experiments, the stimulus consisted of a full-field $\left(12^{\circ}\right.$ vertical by $15^{\circ}$ horizontal $)$ counterphase flickering checkerboard at $8 \mathrm{~Hz}$. This rate was chosen to entrain alpha and beta responses through frequency doubling. A square fixation point was placed in the center of the visual field, and a uniform gray field of the same mean luminance as the checkerboard with a central square fixation point was presented between stimulus presentations. Stimuli had either an abrupt onset and offset (the no-ramp condition), or had onsets and offsets in which the contrast over time followed a raised cosine function (Fig. 1). The definition of stimulus duration for these ramped conditions is the time between $50 \%$ of the maximum contrast. Stimuli had durations of either 1 or $2 \mathrm{~s}$, and had ramp durations of $0,0.5$, or $1 \mathrm{~s}$ in length. Since eyeblink frequency is variable (Doughty, 2001), subjects were allowed to blink at their own comfortable frequency. However, even with online and offline correction, subject eyeblinks create very large artifacts in MEG data. Short stimulus durations were thus chosen to avoid eye blink artifacts from intruding during the stimulus presentation. To maximize the amount of data collected during a run, the inter-stimulus interval (ISI) was $3 \mathrm{~s}$ and defined as the duration between the $50 \%$ of the maximum contrast between stimulus presentations. Ramp and stimulus durations were constant within each acquired 242-s run that was divided into 4- or 5-s epochs. Subjects fixated on a square fixation point placed in the center of the visual field without a task. An MEG session consisted of six runs to examine each combination of the two stimulus durations and three ramp conditions. Each ramp and stimulus duration condition was run once per subject.

B.3. MEG Data Acquisition. During each run, MEG data was collected on five subjects in a 275-channel CTF whole head scanner (Coquitlam, British Columbia, Canada) at a sampling rate of $600 \mathrm{~Hz}$ in a magnetically shielded room (Vacuumschmelze, Germany) with active noise cancellation (i.e., synthetic 3rd gradient balancing).

B.4. MEG Data Analysis. Noise from the acquired MEG data was removed with a $3 \mathrm{rd}$ order gradient. The DC offset and $60-\mathrm{Hz}$ powerline were also subtracted. Data was high-pass filtered at $0.7 \mathrm{~Hz}$. About $500 \mathrm{~ms}$ of data around eye blinks (exceeding 1pT in amplitude and $1 \mathrm{pT} / \mathrm{s}$ ) were computationally identified and removed. Approximately $30 \%$ of the trials were removed due to eye-blink. The stimulus epochs were then averaged within each acquisition for 


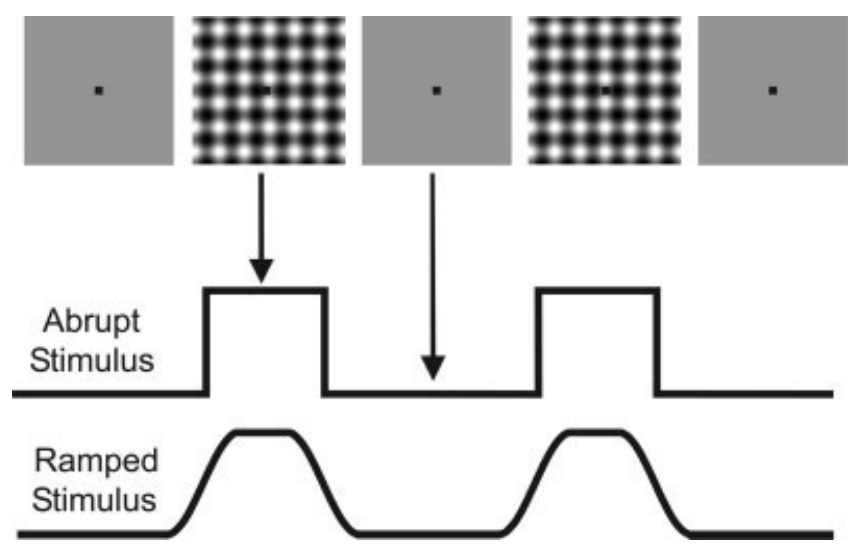

Figure 1. Ramped Paradigm Design. Abrupt on/off transitions were substituted with a ramped transition modeled after a raised cosine function. Gray background presentations were fixed ( 3 s for MEG and $15 \mathrm{~s}$ for $\mathrm{fMRI}$ ) while checkerboard stimulus presentations varied (1 or $2 \mathrm{~s}$ for MEG and 1, 3, 6, or $12 \mathrm{~s}$ for fMRI). Inter-stimulus interval durations were defined as the duration between $50 \%$ of maximum contrast between stimulus presentations. Stimulus duration was defined as the duration between $50 \%$ of maximum contrast.

each channel. The amplitude of the MEG response was converted into the frequency domain by computing the Stockwell transform (or S-transform), from the averaged acquisitions. The Stockwell transform is similar to the Fourier transform with the primary difference that frequency-dependent Gaussian time windows are utilized (Stockwell et al., 1996; Goodyear et al., 2004). This creates a tradeoff between increased temporal resolution at higher frequencies where narrower time windows are used and increased frequency resolution at lower frequencies where wider time windows are used.

\section{C. fMRI Experiment.}

C.1. fMRI Stimulus Presentation Apparatus. Stimuli for the fMRI experiments were generated with the same system and stimulation program used in the MEG experiments. Images were produced using a Sharp PG-210U projector (Camus, WA) fitted with a zoom lens and projected onto a back projection screen. Viewing distance was $367 \mathrm{~cm}$. Projector refresh rate was $60 \mathrm{~Hz}$. During fMRI data acquisition, subjects viewed the image on a screen near the subject's legs through a mirror mounted to the MRI table above the subject's eyes. Subjects' heads were stabilized with a vacuumpack pillow system (S\&S Technology, Houston, TX) system.

C.2. fMRI Stimulus. Stimulus durations were 1, 3, 6, or $12 \mathrm{~s}$ and ramp durations were $0,0.5$, and $1 \mathrm{~s}$ in length. These stimulus durations were chosen to duplicate previous findings (Boynton et al., 1996) and to overlap with at least one of the stimulus durations presented in the MEG stimulus paradigm (i.e., the 1-s stimulus duration). The ISI was $15 \mathrm{~s}$ to allow for a full recovery of the hemodynamic response, and to avoid any hemodynamic or neuronal refractory period. Studies have shown, for example, that the BOLD response exhibits a refractory period of up to $6 \mathrm{~s}$ (Huettel and McCarthy, 2000), and that the nonlinearity of the BOLD response can be altered when the BOLD responses overlap (Birn and Bandettini, 2005). Each fMRI scan lasted $242 \mathrm{~s}$ with ramp and stimulus durations held constant. Each experimental session consisted of 12 stimulus scans (one for each combination of stimulus and ramp duration) followed by an anatomical scan (MPRAGE; $1 \times 1 \times 1 \mathrm{~mm}^{3}$ resolution), using a standard T1-weighted gradient echo pulse sequence. The stimulus scans were presented in random order. During the fMRI scans, subjects fixated on the square fixation point and performed a one-back task comparing stimulus duration. Each subject participated in one experimental session.

C.3.fMRI Data Acquisition. A series of 242 axial T2*-weighted echo planar images (EPI) was acquired on a 3-T GE Signa MR scanner (Waukesha, WI) (TR: $1 \mathrm{~s}$; TE: $30 \mathrm{~ms}$; field of view: $24 \mathrm{~cm}$; slice thickness: $5 \mathrm{~mm}$; $90^{\circ}$ flip angle; matrix size: $\left.64 \times 64\right)$. A brain-specific quadrature Medical Advances RF coil was used (Wauwautosa, WI). A limited coverage of 8-12 slices was used, allowing a TR of $1 \mathrm{~s}$ in order to improve sampling of the hemodynamic response. An additional set of high resolution, T1-weighted, inversion-recovery spoiled gradient-echo anatomic reference images $(\mathrm{TE}=5.3 \mathrm{~ms}, \mathrm{TR}=12 \mathrm{~ms}, \mathrm{TI}=725 \mathrm{~ms}, \mathrm{FOV}=24 \mathrm{~cm}$, matrix $=256 \times 192$, slice thickness $=1.2 \mathrm{~mm}, 17^{\circ}$ flip angle, and 124 axial slices) were obtained for localization purposes.

C.4. fMRI Data Analysis. Data were analyzed by fitting the ideal hemodynamic BOLD response to each pixel's entire signal intensity time course. These ideal time responses were computed by convolving a gamma variate function $h(t)=t^{8.6} e^{-t / 0.547}$ (Cohen, 1997) with stimulus blocks consisting of boxcars of the nominal stimulus duration. Time to peak for the $\gamma$ variate function was $4.7 \mathrm{~s}$ with a full width at maximum height of $3.8 \mathrm{~s}$. This multiple linear regression analysis was performed using Analysis of Functional NeuroImages (AFNI) software, including a regressor to model linear trends, or drifts, in the data (Cox, 1996). Only voxels in visual cortex exceeding a correlation threshold of 0.3 for all combinations of stimulus and ramp duration within a subject were used to define the subject's region of interest (ROI). A voxel which did not exceed this threshold for every combination was excluded from the ROI. While there is spatial heterogeneity of BOLD responses within an ROI (Birn et al., 2001; Pfeuffer et al., 2003), we utilized a relatively large ROI (on average 159 voxels per ROI) for our fMRI analyses to mirror the large ROI used in our MEG analyses. Time courses of voxels in each subject's ROI were averaged for each combination of stimulus and ramp condition. These averaged response time courses were used in a linear systems analysis similar to (Boynton et al., 1996) to test the effects of varying ramp and stimulus durations. In this analysis, the hemodynamic responses to long duration stimuli were predicted by summing copies of appropriately shifted responses to short duration stimuli. The four stimulus durations of $1,3,6$, and 12 s provide six predictions. For example, shifting and adding four responses to a 3-s stimulus was used to predict the response to a 12 -s stimulus.

C.5. Prediction of fMRI Response from MEG Data. MEG response curves for each stimulus and ramp duration were generated by summing the amplitudes of the Stockwell Transformation in the 0 - to $40-\mathrm{Hz}$ range. Estimates of the MEG response to longer stimulus durations of 3,6 , and $12 \mathrm{~s}$ were obtained by extending the steady-state period (filling in a value equal to the average amplitude during this steady state), and using for the ramp periods an average of the response during the ramps from the 1- and 2-s stimuli. Each of these MEG response curves was convolved with a $\gamma$ variate function to represent an ideal hemodynamic BOLD response. These convolved predictions were compared with the fMRI data.

The degree of nonlinearity for both the measured BOLD response and the MEG predicted BOLD response was computed by fitting ideal response functions (a $\gamma$ variate convolved with a boxcar of width equal to the nominal stimulus duration) to either the measured or predicted BOLD response for each stimulus duration, using 

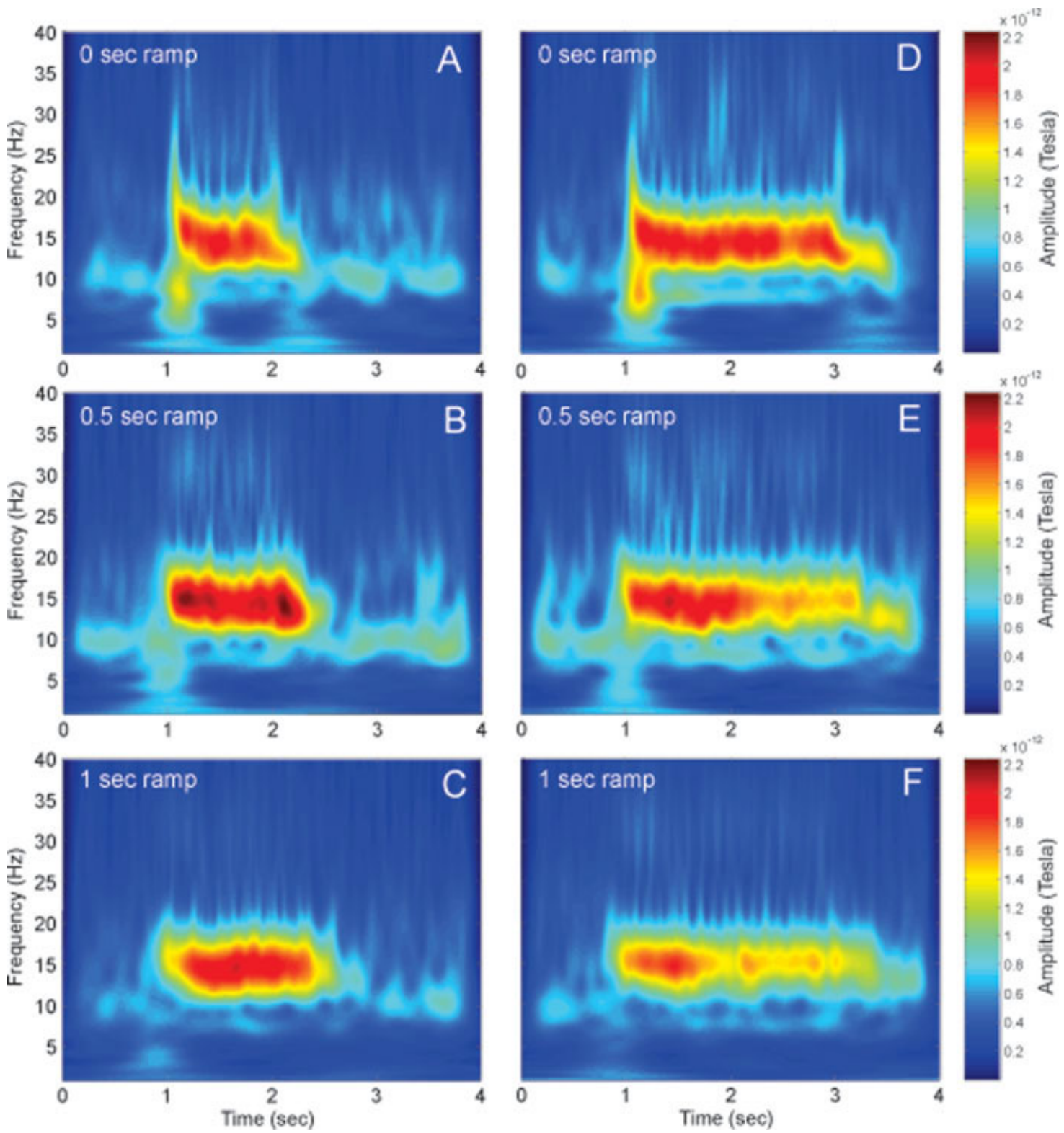

Figure 2. Stockwell Transformation of MEG responses. Time-frequency plots created using a Stockwell transformation on our averaged MEG recordings in occipital sensors. The time window decreases with frequency. The left column $(\mathrm{A}-\mathrm{C})$ shows responses to a stimulus of $1 \mathrm{~s}$ in duration while the right column (D-F) shows the response to a 2-s stimulus. A transient onset response in the 5- to $30-\mathrm{Hz}$ range can be seen at stimulus onset in the no-ramp condition while a reduction of the transient onset response is observed in the 1-s ramp condition. The 0.5-s ramp condition shows a moderate reduction in transient onset response. The color bar to the right of each figure shows the amplitude of the response (in units Tesla).

a least squares method. The resulting fit coefficients (which scaled the predicted response to the observed response) were normalized by the amplitude of the fit to the longest (12 s) duration response. If the relationship between the stimulus and the MEG response was linear, then the fit coefficients of the ideal responses should be the same for all stimulus durations, and the degree of nonlinearity should be equal to 1 . Finally, the linearity of the MEG-predicted BOLD response was also assessed by superposition, to allow a direct comparison with the analysis performed for the fMRI study. This comparison can show how much of the nonlinearity evident in the BOLD response can be explained by the neuronal transients, as measured with MEG.

\section{RESULTS}

A. MEG Experiment. We first examined the ramp paradigm with MEG because it offers the high temporal resolution necessary to determine if a ramped onset reduces the transient response. A counterphase-modulated stimulus induces a frequency-doubled MEG and EEG signal. This can be seen in the Stockwell transformation (Fig. 2), which shows a strong $16-\mathrm{Hz}$ response to the $8-\mathrm{Hz}$ stimulus. Figure 2 shows the average of the Stockwell transformed MEG responses averaged across all occipital channels for all subjects. As expected, the predominant response for each ramp and stimulus duration occurs at $16 \mathrm{~Hz}$. Robust transient responses near the onset of the stimuli in the no-ramp condition are evident for both stimulus 

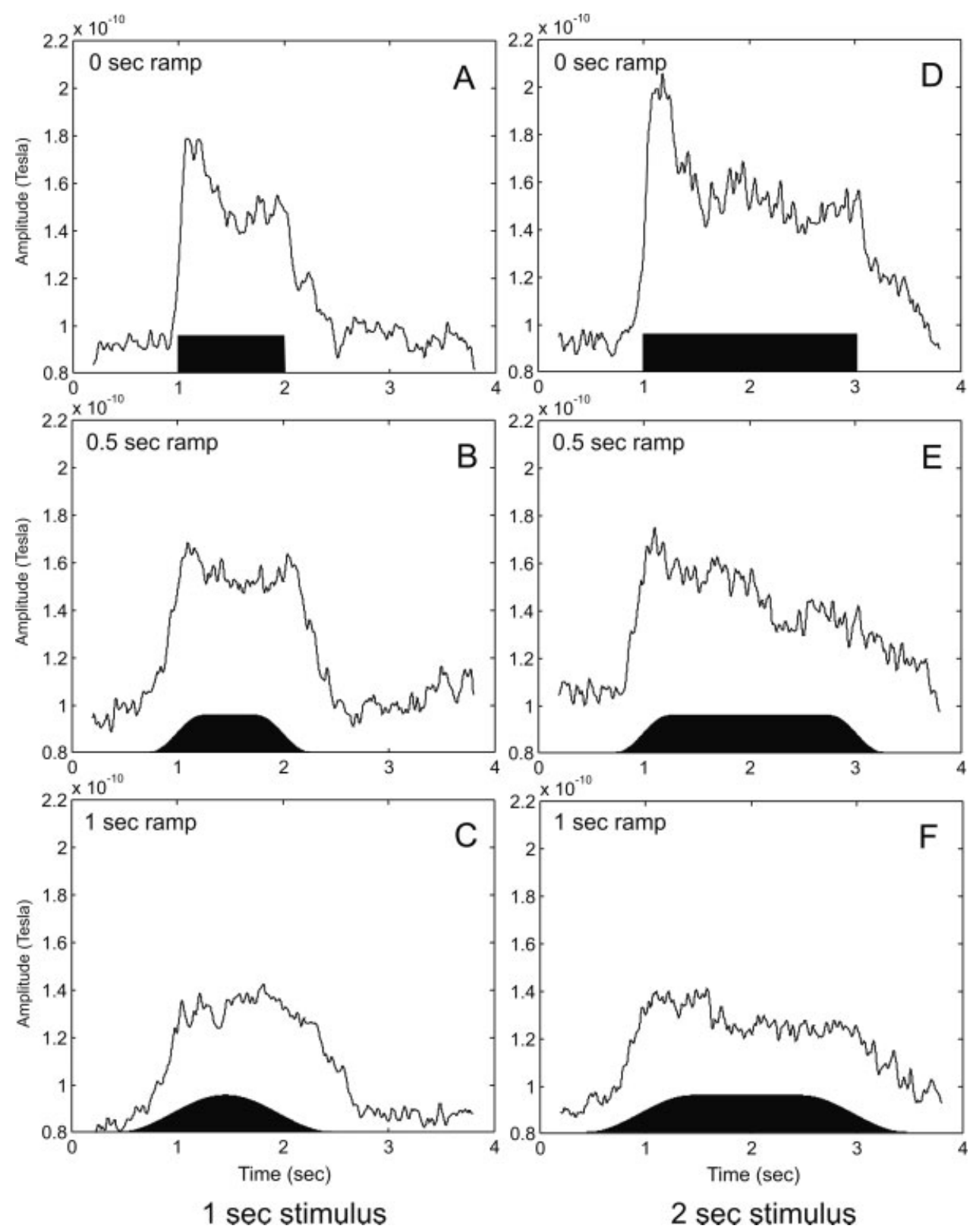

Figure 3. Summed amplitudes of the Stockwell Transform. The amplitudes in the 0- to 40-Hz range from the Stockwell Transformation in Figure 2 are summed. Below each summed response is the maximum stimulus contrast as a function of time. The left column shows responses to a stimulus of $1 \mathrm{~s}$ in duration while the right column shows the response to a 2-s stimulus. A transient onset response can be seen at stimulus onset in the no-ramp condition while a reduction of the transient onset response is observed with increasing ramp duration.

durations of 1 and $2 \mathrm{~s}$ (Figs. 2A and 2D). This is in agreement with a previous MEG study showing transient onset response in the 5- to $10-\mathrm{Hz}$ range (Fawcett et al., 2004). In the 0.5- and 1-s ramp condition (Figs. 2B-2C, 2E-2F), the transient response is reduced regardless of stimulus duration. The longer ramp duration shows the greatest reduction in transient response. The steady state response to the stimulus appears to be the same across all ramp durations.

Figure 3 shows the summed amplitude of the Stockwell transformation in the 0 - to $40-\mathrm{Hz}$ range. For both 1 - and 2 -s stimulus durations, the summed amplitudes show robust transient onset responses for the no-ramp condition and a reduction in transient response in the 1-s ramp stimulus. The MEG response to a 1-s ramped stimulus shows a rapid increase during the ramp, reaching steady state levels after only a relatively small change in contrast. Steady state responses are similar in all six conditions. This produces a similar pattern of transient response attenuation as seen in the Stockwell transformations and suggests that the ramped stimulus paradigm reduces the transient onset response across all frequencies as measured by MEG. These findings demonstrate that varying the duration of a ramped onset and offset may modulate and attenuate the transient MEG response.

B. fMRI Experiment. We tested the ramp paradigm's effect on the linearity of the fMRI response by examining whether responses to long duration stimuli could be predicted by short duration stimuli 

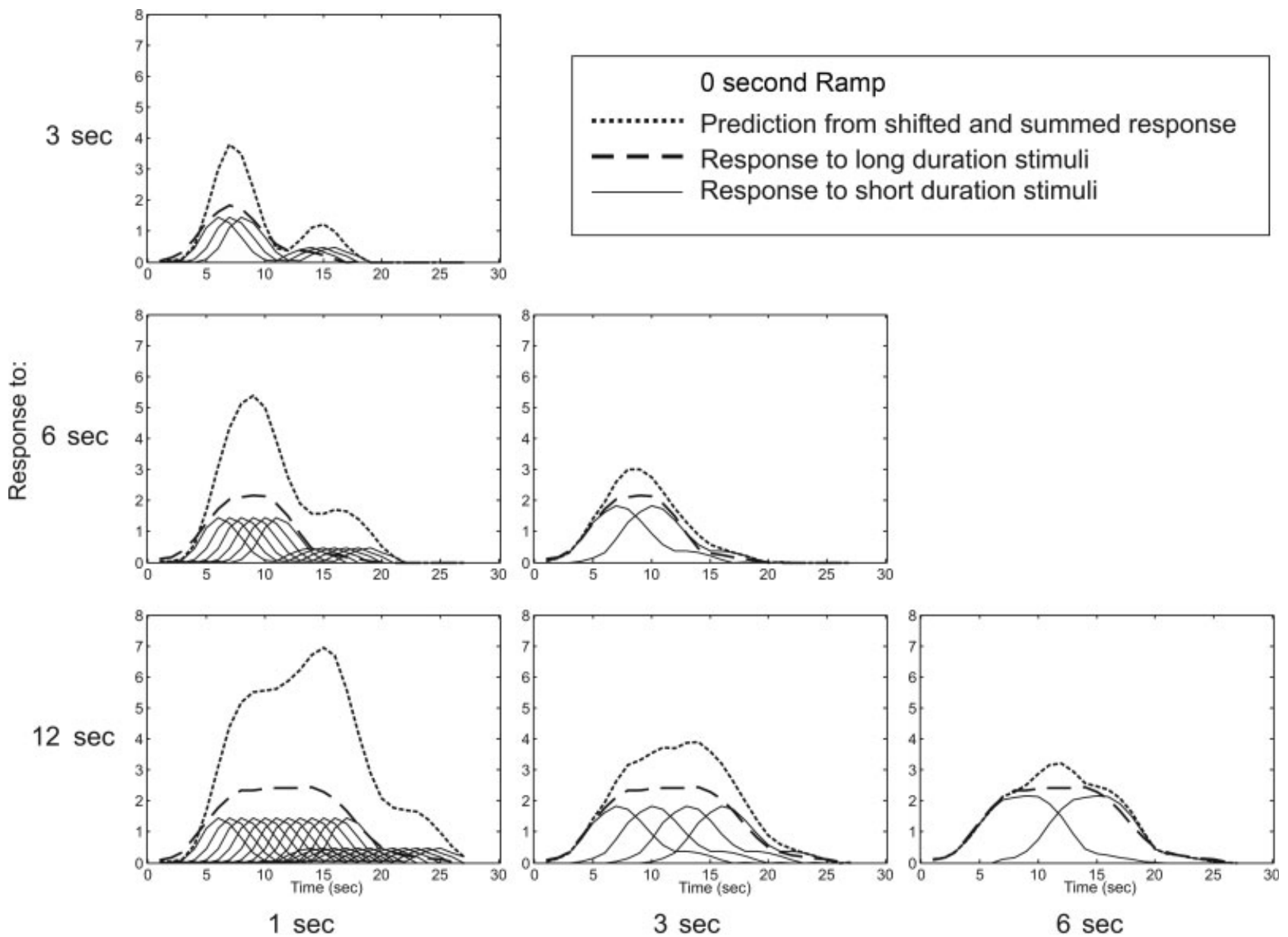

Predicted Response to:

Figure 4. Tests of Superposition. Responses to longer duration stimuli (long dashes) are predicted by superposition of responses to shorter duration stimuli (short dashes). The shifted copies of the response to the shorter duration stimuli are shown in solid lines. Figure 4 shows predictions using no ramp, fMRI responses to 1,3 , and $6 \mathrm{~s}$ were used to predict the response to 3,6 , and $12 \mathrm{~s}$. The system becomes more linear with longer durations, but a large nonlinearity occurs with short duration stimuli $(1 \mathrm{~s})$. Utilizing a 0.5 - or 1 -s ramp does not appreciably reduce the nonlinearity at short durations.

(Boynton et al., 1996). Figure 4 shows the results of the predictions in the no-ramp condition. The shifted copies of the response to the shorter duration stimulus are shown with solid lines, the response to the longer duration stimulus is shown with the long dashed line, and the prediction is shown with the short dashed line. This replicates the original examination of linearity in fMRI (Boynton et al., 1996) but with additional shorter duration stimuli, as well as Vazquez and Noll's work where they demonstrated increasing nonlinear behavior with short duration stimuli when using a sinusoidally ramped onset and offset. Consistent with previous studies (Boynton et al., 1996; Vazquez and Noll, 1998), the fMRI response is approximately linear when the 6-s stimulus is used to predict the 12-s response, but diverges from linearity when predicting with short stimulus durations ( 1 and $3 \mathrm{~s}$ ). The overprediction becomes increasingly exaggerated when predicting longer stimulus durations.

Figures 5 and 6 show the same analysis for the 0.5 - and 1-s ramp conditions, respectively. The predictions of the fMRI responses in these conditions are remarkably similar to the no-ramp condition, showing a disproportionately large response with shorter duration stimuli with a tendency towards a linear approximation of the fMRI response signal with longer duration stimuli. This trend is consistent for all six comparisons. The similarity of the fMRI results across ramp durations is quite striking in light of how increasing the ramp duration attenuates the MEG transient response. The linearity of the fMRI response is not modulated by the duration of that onset and offset ramp in stimulus contrast.

C. A Different Definition of Stimulus Duration. The analysis of nonlinearity in the fMRI data depends critically on the duration of the stimulus and the resulting neuronal activity. The precise duration of a ramped stimulus depends on its definition. In this study, the duration of the stimulus was defined as the time between $50 \%$ contrast because with raised cosine ramps, the superposition analysis is valid with respect to the physical stimulus. For example, three shifted copies of a 1-s ramped stimulus will add perfectly to form a single 3-s stimulus.

However, as known from earlier studies and evident from our MEG data, the neuronal response is not necessarily a linear function of contrast; neuronal and fMRI responses at lower contrasts are disproportionately larger than at higher contrasts (Miller et al., 1980; 

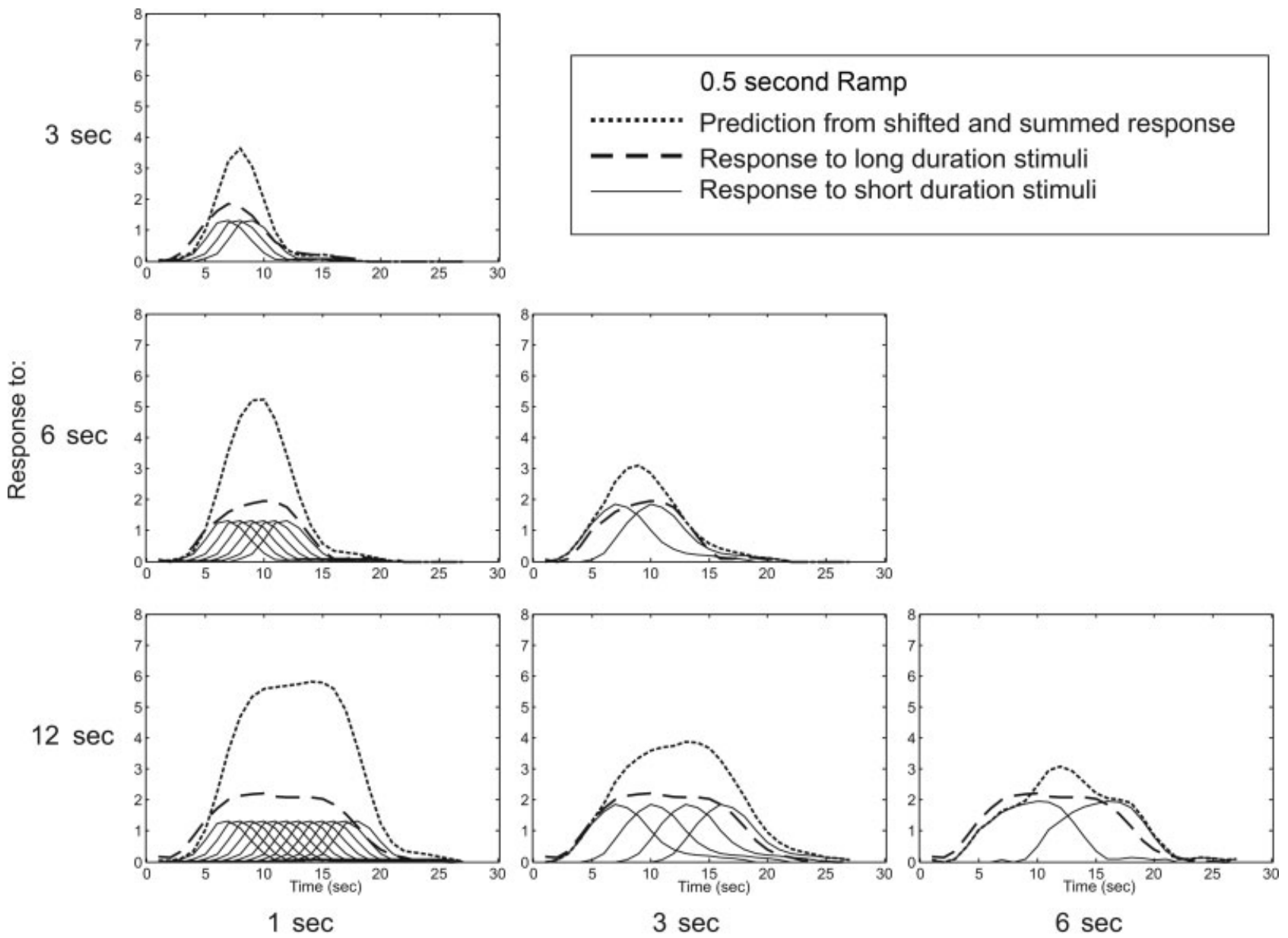

Predicted Response to:

Figure 5. Tests of Superposition. Responses to longer duration stimuli (long dashes) are predicted by superposition of responses to shorter duration stimuli (short dashes). The shifted copies of the response to the shorter duration stimuli are shown in solid lines. Figure 5 shows predictions using a 0.5 -s ramp, fMRI responses to 1,3 , and $6 \mathrm{~s}$ were used to predict the response to 3,6 , and $12 \mathrm{~s}$. The system becomes more linear with longer durations, but a large nonlinearity occurs with short duration stimuli $(1 \mathrm{~s})$. Utilizing a 0.5 - or 1 -s ramp does not appreciably reduce the nonlinearity at short durations.

Boynton et al., 1996; Boynton et al., 1999; Seidemann et al., 1999; Logothetis et al., 2001). So while the ramp may reduce the transient overshoot activity relative to the steady state, it may also effectively increase the duration of the neuronal response. Our initial test for superposition may therefore not yield a fair prediction because although the physical stimuli may shift and add appropriately, the neuronal responses may not.

We therefore conducted a second test of superposition by assuming the extreme case of defining the stimulus duration to include the entire ramp duration (Fig. 7). A 1-s stimulus with a 0.5 -s ramp duration was therefore considered to have a duration of $1.5 \mathrm{~s}$, so that only two shifted copies of the response were used to predict a 3-s stimulus. The neuronal activity in this case is assumed to be firing at its steady-state level for the duration of the stimulus, including the ramp, which is likely to be an overestimation of any nonlinear relationship between stimulus contrast and neuronal response. This analysis should therefore skew the prediction to underestimate the response to the longer stimulus duration.

Figure 8 shows the results of the predictions when utilizing this new definition of stimulus duration. Only comparisons between conditions which shared the same ramp condition and whose longer stimulus duration was a multiple of the shorter stimulus duration were examined. Even with this extreme definition of stimulus duration, we still see an overprediction of the response to longer stimuli from shorter stimulus durations. That is, even though we are now summing fewer responses to generate the predicted response, we still observe a disproportionately large fMRI response to short duration stimuli. This overprediction from short stimulus durations, after accounting for a possible lengthening of the response, is strong evidence that our fMRI response to the stimulus remains nonlinear with the ramp.

D. Predicting the fMRI Response from MEG Data. Another way to compare the results across methodologies is to directly predict the fMRI response from the MEG response by convolving the total MEG output in the 0 - to $40-\mathrm{Hz}$ range with a hemodynamic impulse response function. Each combination of stimulus and ramp duration was convolved separately. Figure 9 shows the actual and predicted fMRI response to different ramp and stimulus durations. Qualitatively, the MEG-derived fMRI response does not appear to 

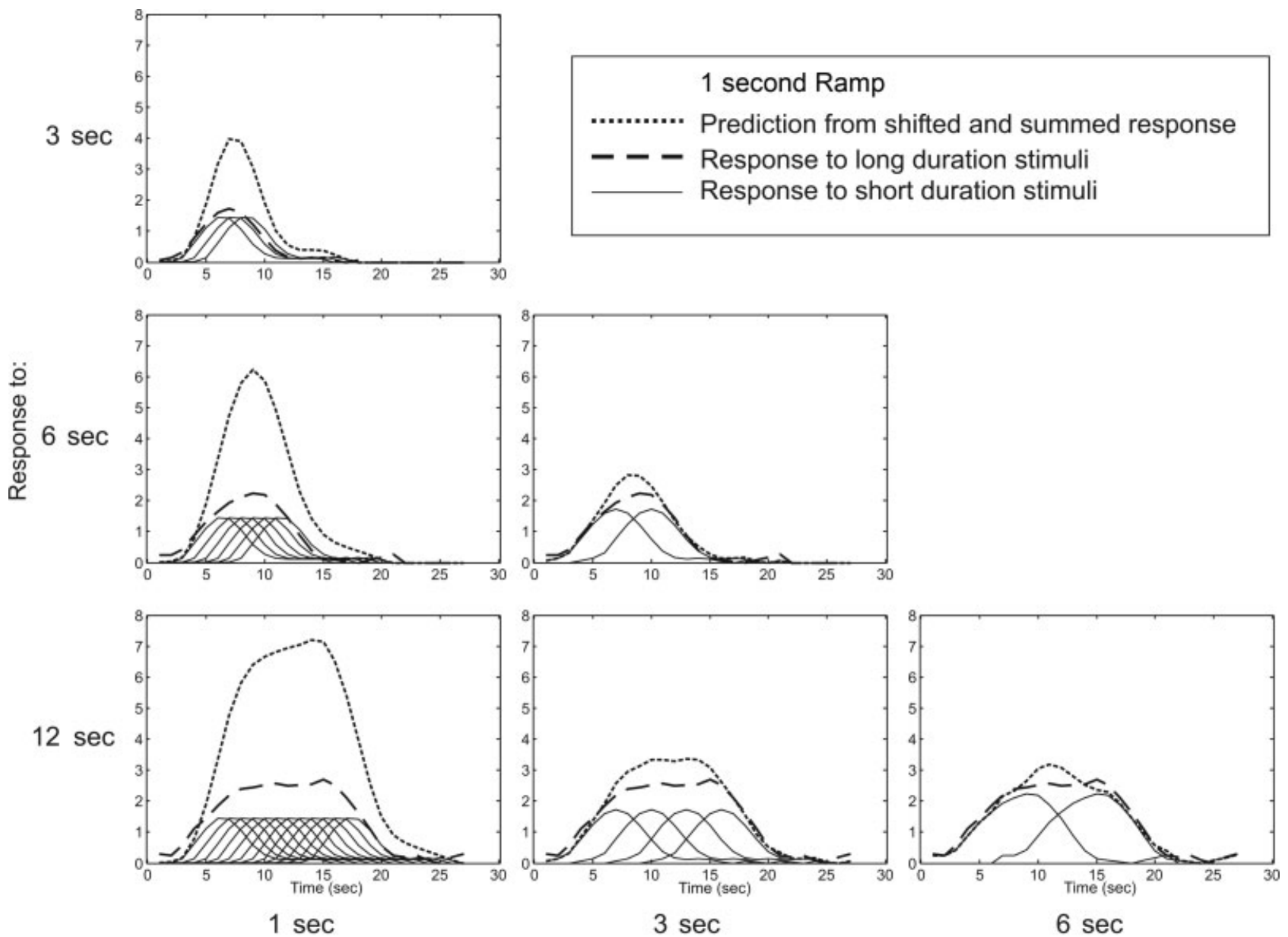

Predicted Response to:

Figure 6. Tests of Superposition. Responses to longer duration stimuli (long dashes) are predicted by superposition of responses to shorter duration stimuli (short dashes). The shifted copies of the response to the shorter duration stimuli are shown in solid lines. Figure 6 shows predictions using a 1-s ramp. fMRI responses to 1,3 , and $6 \mathrm{~s}$ were used to predict the response to 3 , 6 , and $12 \mathrm{~s}$. The system becomes more linear with longer durations, but a large nonlinearity occurs with short duration stimuli $(1 \mathrm{~s})$. Utilizing a 0.5 - or 1 -s ramp does not appreciably reduce the nonlinearity at short durations.

show the large nonlinearity at shorter stimulus durations that exists in the fMRI data. Figures $10 \mathrm{~A}$ and $10 \mathrm{~B}$ shows the regression analysis of fitting the ideal responses (based on a simple boxcar stimulus convolved with a $\gamma$-variate function) to the data. With longer stimulus durations, the fits approach unity with both the BOLD data and the MEG-derived BOLD predictions, but with the shortest stimulus duration (1 s), there is a deviation from unity. The BOLD data at
1 s shows a nonlinearity of $\sim 2.5$-fold relative to the ideal fit while the MEG-derived prediction has a nonlinearity of $\sim 1.5$-fold. The slight nonlinearity of the MEG response to the stimulus is due to an overshoot in the neuronal activity relative to the steady state for the 0 - and 0.5 -s ramp durations, and to an increased duration of the neuronal response (relative to the nominal stimulus duration) for the 0.5 - and 1-s ramp durations. While the MEG-derived BOLD
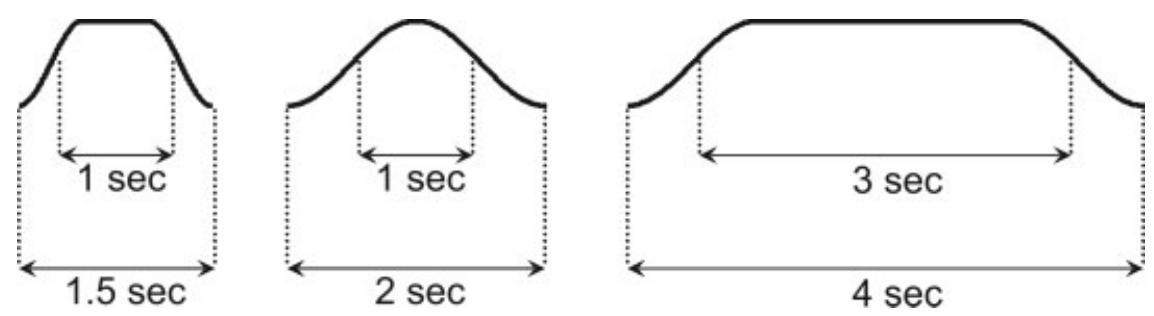

Figure 7. Alternate definition of stimulus duration. The initial definition of stimulus duration was the width of the stimulus at half-maximum of its contrast (shown in the top set of arrows). The alternative, or 'strict,' definition of stimulus duration includes the entirety of the ramp onset and offset durations (shown in the bottom set of arrows). 

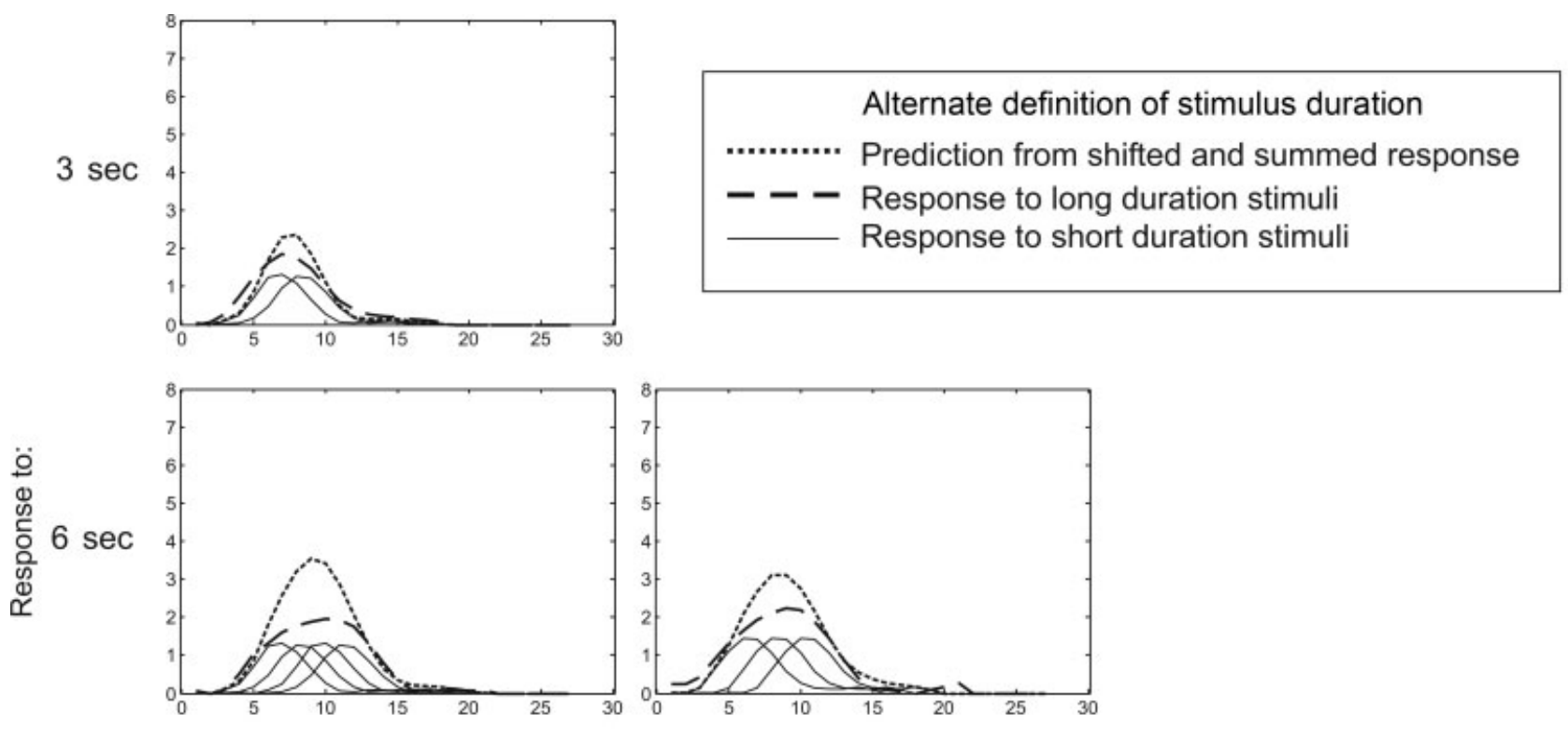

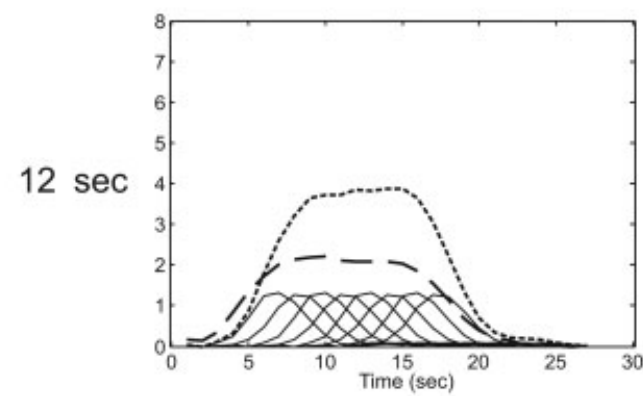

$1 \mathrm{sec}$ stimulus, $0.5 \mathrm{sec}$ Ramp

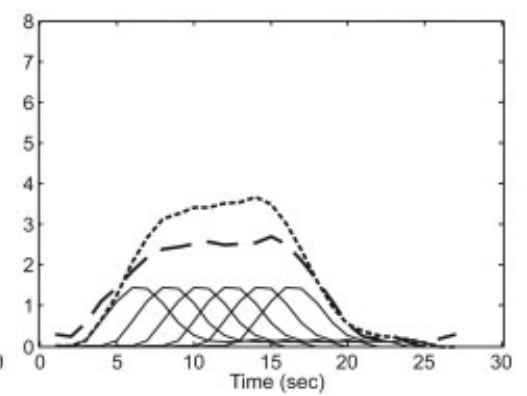

$1 \mathrm{sec}$ stimulus, $1 \mathrm{sec}$ Ramp

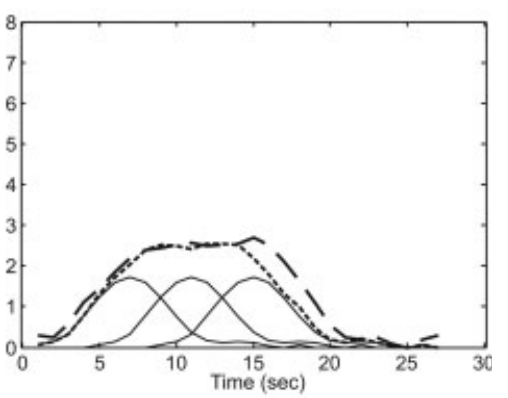

3 sec stimulus, $1 \mathrm{sec}$ Ramp

Predicted Response to:

Figure 8. Test of superposition using an alternative definition of stimulus duration. Using a more conservative yet skewed interpretation of stimulus duration still generates an overprediction at short duration stimuli. Stimulus duration includes the ramp durations and is defined as the duration from stimulus onset to offset. In all cases except for one, a longer response (3, 6, or $12 \mathrm{~s})$ was predicted by the fMRI response to a 1-s stimulus duration (as defined by the old definition) with the same ramp duration as the longer response. In the bottom right figure, the comparison is with a 3-s stimulus duration and 1-s ramp predicting the response to a 12-s stimulus with the same ramp duration.

prediction demonstrates a disproportionately large increase at short stimulus durations, it is dwarfed by the BOLD response's nonlinearity.

Since the MEG response to the 1- and 2-s stimulus durations with a 1-s ramp appeared similar to boxcar functions of 2- and 4-s durations, respectively, the linearity was also assessed by summing two copies of the MEG-predicted BOLD response to a 1-s stimulus and comparing this to the MEG-predicted BOLD response for a 3-s duration stimulus (Fig. 10). This is analogous to the "extreme" case shown in Figure 8, which as discussed earlier clearly illustrates the continued overprediction even when fewer responses are summed to create the predicted response. In this case, however, the MEG-derived fMRI responses show an underprediction of the longer stimulus duration when using a different definition of the stimulus duration. The convolved response to a stimulus of $3 \mathrm{~s}$ in length is larger than the summed and shifted responses to two stimuli that are $1 \mathrm{~s}$ in length. This again demonstrates that the nonlinearity measured in BOLD responses is much larger than would be predicted based on MEG responses.

\section{DISCUSSION}

The fMRI response has been shown to be disproportionately large for short stimulus durations (Boynton et al., 1996; Dale and Buckner, 1997; Vazquez and Noll, 1998). This nonlinearity has been shown to vary considerably across space with a given voxel showing an eight-fold over-prediction while a nearby voxel had only a three-fold nonlinearity (Birn et al., 2001). While one group has explored the possibility of hemodynamic transients through simulations (Obata et al., 2004), no previous study has directly examined the degree to which transients in the neuronal response can account for the observed BOLD nonlinearity, and how the nonlinearity is affected when these transients are modulated.

In the first part of our study, we took advantage of the high temporal resolution of MEG and found that the MEG transient onset and offset response is reduced with the presentation of a ramped onset and offset instead of an abrupt transition in contrast. We also observed that the transient response as measured with MEG is reduced with increasing ramp durations. However, our fMRI responses to the ramped paradigm showed no change in the 


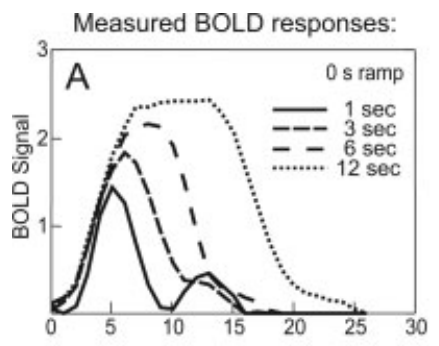

Predicted responses from MEG:
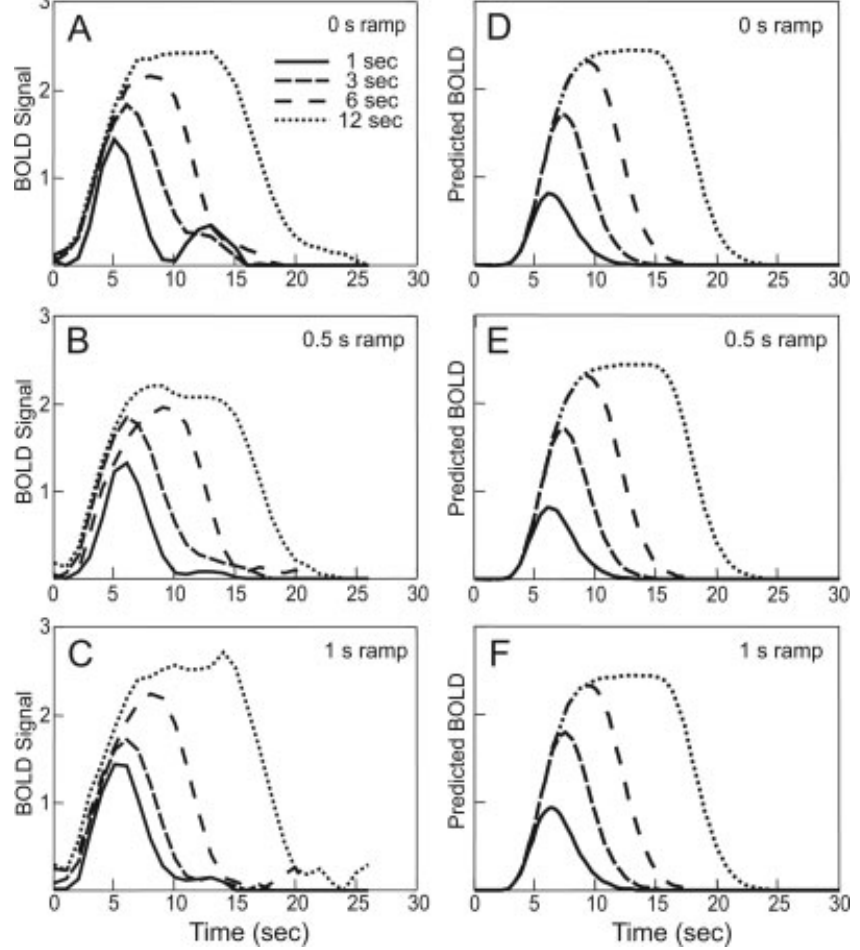

Figure 9. Predicting $\mathrm{fMRI}$ responses from MEG Data. Time courses of $\mathrm{fMRI}$ signal to different stimulus durations and ramp durations (AC). Predicted $\mathrm{fMRI}$ responses to the same conditions using MEG signal (D-F). The MEG responses are convolved with a hemodynamic response filter to generate the predicted responses. $\mathrm{fMRI}$ responses to longer duration were generated by extending the steady-state MEG response to shorter durations then convolving with the hemodynamic response filter.

nonlinearity of the fMRI signal to short duration stimuli. Modulating the duration of the ramped onset and offset also did not influence the amount of nonlinearity in the fMRI signal, with responses to the 1-s stimuli showing a 2.5-fold over-prediction. In comparison, using the MEG responses as surrogates for neuronal responses, the 1-s stimulus shows only a 1.4 -fold over-prediction. This means that the amount of the nonlinearity that can be explained by the neuronal transients measured by MEG is not sufficient to account for all of the nonlinearity in the BOLD response, explaining only about half of the observed overshoot. These results suggest that the fMRI nonlinearity in the fMRI response is not solely due to the transient neuronal activity. The source of this additional nonlinearity must therefore either be hemodynamic in origin or the result of other neuronal effects not accounted for in the MEG measurement.

One possible vascular explanation is that a minimal increase in neuronal activity could lead to a dramatic increase in blood flow. Logothetis (2001) showed that local field potentials (LFP) and multi-unit activity (MUA) increased with stimulus contrast at a slower rate then the fMRI response. They demonstrated that at $12.5 \%$ contrast, the steady-state fMRI response was already at $50 \%$ of maximum amplitude while LFP and MUA activity was only about $20 \%$ of maximum. There therefore appears to be a relatively large BOLD signal associated with a small amount of neuronal activity.
The larger than expected responses to brief stimuli may also be the result of a nonlinear relation between other hemodynamic factors, such as the relationship between the oxygen extraction fraction, the cerebral blood flow, and the cerebral blood volume (Vazquez and Noll, 1998; Friston et al., 2000; Obata et al., 2004). A recent work examining the BOLD response to different stimulus duty cycles and stimulus "off" periods, however, has shown that only about half of the observed nonlinearity could be predicted from hemodynamic models of nonlinearity with physiologically plausible parameters (Birn and Bandettini, 2005), consistent with the findings in the present study.

The spatial heterogeneity of the regions of interest (ROIs) used in our MEG and BOLD analysis may also have an influence in the comparison of our two results. In fMRI, the amount of nonlinearity has been shown to be spatially dependent, with some areas of the visual cortex being more nonlinear than others (Birn et al., 2001). fMRI ROIs were restricted to voxels responding to stimuli in all conditions, whereas in MEG, the "ROI" consisted of all the occipital sensors. Although both analyses presumably reflect a population response, the ROIs in the two methods may reflect responses in different spatial locations.

In this study, we used the total power of the MEG signal to model the neuronal activity associated with the BOLD fMRI signal. This choice was motivated in part by earlier MEG studies (e.g., Fawcett et al., 2004) which showed that the strongest MEG response to a oscillating visual stimulus occurred at twice the stimulus frequency and the onset response occurred primarily in the alpha
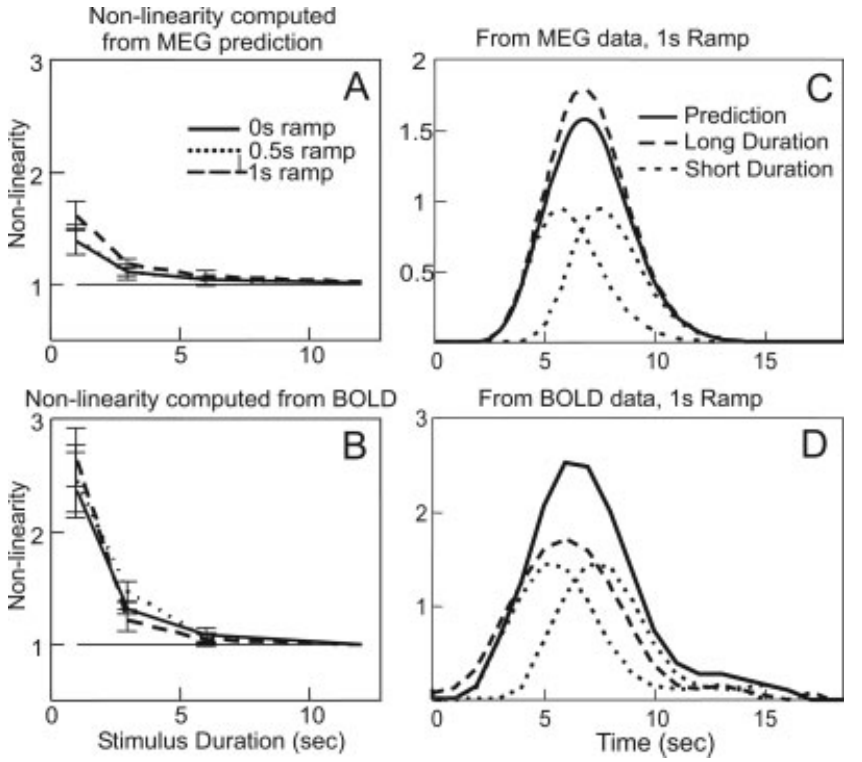

Figure 10. Nonlinearity in MEG-derived and BOLD responses. Fitting the actual and predicted fMRI time courses to an ideal response (where the response to the 12-s stimuli is assumed to be linear in A and $B$ ) shows a deviation from linearity with short stimulus durations. While we observe an over response in the MEG-derived predicted time courses (A), we see a larger nonlinearity in the BOLD time courses (B). Assuming the alternative definition of stimulus duration, we observe the predicted response (solid line) from shorter responses is smaller than the response to the long duration stimulus (long dashed line) in the MEG-derived time series (C). However, using this alternative definition, the predicted response (solid line) is much larger than the response to the long duration stimulus (long dashed line) in the BOLD time courses (D). 
band $(\sim 10 \mathrm{~Hz})$. Our data showed a very similar pattern, with the strongest fluctuations occurring at twice the stimulus frequency (i.e., at $16 \mathrm{~Hz}$ ), and the onset transient occurring in a broader frequency band, between 5 and $30 \mathrm{~Hz}$. No frequency bands showed a decrease in power correlated with the stimulus timing. In fact, there is a growing body of literature demonstrating that both low (delta) and high (gamma) frequencies correlate positively with BOLD signal increases (Niessing et al., 2005; Lu et al., 2007). Since our analysis focuses on the evoked responses, which are less sensitive to higher frequencies (e.g., induced beta or gamma), there is a possibility that we are underestimating the amplitude of the transient. However, since the power of the MEG signal generally scales as $1 /$ $\mathrm{f}$, we expect that the contribution of the induced beta or gamma to the total power, particularly for this oscillatory visual stimulus, to be small.

An additional reason for the difference in the nonlinearity predicted by MEG and observed in BOLD is that the measured MEG signal may not capture all of the neuronal activity in the cortex. The magnetic fields generated by the synchronous activity of pyramidal cortical neurons oriented perpendicular to the surface of scalp, for example, are generally not detected with MEG. However, to explain a change in the linearity, i.e., a change in the ratio of the transient to steady-state response, disproportionately more neurons that respond transiently to the stimulus must be undetected. This would only be possible if neurons which convey transient information during the ramp phase of the stimulus lie in an orientation perpendicular to the surface of the scalp, and are therefore undetected, while neurons responding to the steady-state response lie in an orientation more parallel to the scalp. In this case, one could use EEG to determine the existence of this population of perpendicularly-oriented neurons. The scenario of transient neurons lying in pooled groups of different orientations for different ramp durations seems highly unlikely. A decreased ability to detect neuronal activity by MEG can also result from a reduction in phase coherence among neurons. In this case, the magnetic fields sum incoherently and cancel each other, resulting in a decreased MEG response but with possibly no change in the neuronal metabolism and associated BOLD fMRI response. It is also possible that a reduction in phase coherence due to the gradual activation of neurons to different ramp stimuli is occurring as well. In order to explain a change in the linearity, a greater phase incoherence coupled with increased metabolism must be present for brief stimuli and during the ramps in stimulus contrast.

\section{v. CONCLUSION}

In summary, our MEG measurements show that the abrupt onset of a visual stimulus induces a transient overshoot in neuronal activity. These transients are consistent with the observation that fMRI responses to shorter stimulus durations are larger than expected from a linear convolution of the stimulus time-course with a hemodynamic response function. Indeed, BOLD signals predicted from our MEG responses to abrupt onset stimuli do show disproportionately large responses to short stimulus durations, but these predictions under a linear hemodynamic response, while accounting for neuronal nonlinearities, are not as large as that observed in our fMRI measurements. The transient response in the MEG signal is reduced when the contrast is ramped up slowly. This reduces the predicted BOLD signal. However, the measured BOLD response to short duration ramped stimuli remains disproportionately large.

Since the steady-state neuronal response is a decelerating function of stimulus contrast, our ramped stimulus effectively lengthens the duration of the neuronal response. If the contrast-response curve is taken into account by assuming a longer duration neuronal response, then the MEG response for a 1-s ramp behaves more linear, while the BOLD response remains nonlinear. The neuronal overshoot with abrupt onset stimuli measured by MEG can only explain about half of the nonlinearity observed in the BOLD response. These results suggest that the nonlinearity of the fMRI response is not solely due to transient activity, and that approximately half of it must be accounted for by other neuronal or vascular contributions.

\section{ACKNOWLEDGMENTS}

The authors thank Fred Carver and Tom Holroyd for their assistance in MEG data acquisition and analysis. They also thank their anonymous reviewer for constructive criticism and suggestions on improving this manuscript.

\section{REFERENCES}

D.G. Albrecht, S.B. Farrar, and D.B. Hamilton, Spatial contrast adaptation characteristics of neurones recorded in the cat's visual cortex, J Physiol (Lond) 347 (1984), 713-739.

P.A. Bandettini, E.C. Wong, R.S. Hinks, R.S. Tikofsky, and J.S. Hyde, Time course EPI of human brain function during task activation, Magn Reson Med 25 (1992), 390-397.

J.W. Belliveau, D.N. Kennedy Jr., R.C. McKinstry, B.R. Buchbinder, R.M. Weisskoff, M.S. Cohen, J.M. Vevea, T.J. Brady, and B.R. Rosen, Functional mapping of the human visual cortex by magnetic resonance imaging, Science 254 (1991), 716-719.

R.M. Birn and P.A. Bandettini, The effect of stimulus duty cycle and "off" duration on BOLD response linearity, Neuroimage 27 (2005), 70-82.

R.M. Birn, Z.S. Saad, and P.A. Bandettini, Spatial heterogeneity of the nonlinear dynamics in the FMRI BOLD response, Neuroimage 14 (2001), 817826.

A.B. Bonds, Temporal dynamics of contrast gain in single cells of the cat striate cortex, Vis Neurosci 6 (1991), 239-255.

G.M. Boynton, S.A. Engel, G.H. Glover, and D.J. Heeger, Linear systems analysis of functional magnetic resonance imaging in human V1, J Neurosci 16 (1996), 4207-4221.

G.M. Boynton, J.B. Demb, G.H. Glover, and D.J. Heeger, Neuronal basis of contrast discrimination, Vis Res 39 (1999), 257-269.

D.H. Brainard, The psychophysics toolbox, Spat Vis 10 (1997), 433-436.

A.F. Cannestra, N. Pouratian, M.H. Shomer, and A.W. Toga, Refractory periods observed by intrinsic signal and fluorescent dye imaging, J Neurophysiol 80 (1998), 1522-1532.

M.S. Cohen, Parametric analysis of fMRI data using linear systems methods, Neuroimage 6 (1997), 93-103.

R.W. Cox, AFNI: Software for analysis and visualization of functional magnetic resonance neuroimages, Comput Biomed Res 29 (1996), 162-173.

A.M. Dale and R.L. Buckner, Selective averaging of rapidly presented individual trials using fMRI, Hum Brain Mapp 5 (1997), 329-340.

O. David, D. Cosmelli, D. Hasboun, and L. Garnero, A multitrial analysis for revealing significant corticocortical networks in magnetoencephalography and electroencephalography, Neuroimage 20 (2003), 186-201.

M.J. Doughty, Consideration of three types of spontaneous eyeblink activity in normal humans: During reading and video display terminal use, in primary gaze, and while in conversation, Opt Vis Sci 78 (2001), 712-725.

I.P. Fawcett, G.R. Barnes, A. Hillebrand, and K.D. Singh, The temporal frequency tuning of human visual cortex investigated using synthetic aperture magnetometry, Neuroimage 21 (2004), 1542-1553. 
M.D. Fox, A.Z. Snyder, M.P. McAvoy, D.M. Barch, and M.E. Raichle, The BOLD onset transient: Identification of novel functional differences in schizophrenia, Neuroimage 25 (2005a), 771-782.

M.D. Fox, A.Z. Snyder, D.M. Barch, D.A. Gusnard, and M.E. Raichle Transient BOLD responses at block transitions, Neuroimage 28 (2005b), 956966.

K.J. Friston, Imaging neuroscience: Principles or maps? Proc Natl Acad Sci USA 95 (1998), 796-802.

K.J. Friston, A. Mechelli, R. Turner, and C.J. Price, Nonlinear responses in fMRI: The Balloon model, Volterra kernels, and other hemodynamics. Neuroimage 12 (2000), 466-477.

G.H. Glover, Deconvolution of impulse response in event-related BOLD fMRI, Neuroimage 9 (1999), 416-429.

B.G. Goodyear, H. Zhu, R.A. Brown, and J.R. Mitchell, Removal of phase artifacts from fMRI data using a Stockwell transform filter improves brain activity detection, Magn Reson Med 51 (2004), 16-21.

R. Hari and N. Forss, Magnetoencephalography in the study of human somatosensory cortical processing, Philos Trans R Soc Lond B Biol Sci 354 (1999), 1145-1154.

D.J. Heeger and D. Ress, What does fMRI tell us about neuronal activity?, Nat Rev Neurosci 3 (2002), 142-151.

S.A. Huettel and G. McCarthy, Evidence for a refractory period in the hemodynamic response to visual stimuli as measured by MRI, Neuroimage 11 (2000), 547-553.

S.A. Huettel and G. McCarthy, Regional differences in the refractory period of the hemodynamic response: An event-related fMRI study, Neuroimage 14 (2001), 967-976.

S. Inan, T. Mitchell, A. Song, J. Bizzell, and A. Belger, Hemodynamic correlates of stimulus repetition in the visual and auditory cortices: An fMRI study, Neuroimage 21 (2004), 886-893.

K.K. Kwong, J.W. Belliveau, D.A. Chesler, I.E. Goldberg, R.M. Weisskoff, B.P. Poncelet, D.N. Kennedy, B.E. Hoppel, M.S. Cohen, R. Turner, H. Cheng, T. Brady, and B.R. Rosen, Dynamic magnetic resonance imaging of human brain activity during primary sensory stimulation, Proc Natl Acad Sci USA 89 (1992), 5675-5679.

N.K. Logothetis, J. Pauls, M. Augath, T. Trinath, and A. Oeltermann, Neurophysiological investigation of the basis of the fMRI signal, Nature 412 (2001), 150-157.

H. Lu, Y. Zuo, H. Gu, J.A. Waltz, W. Zhan, C.A. Scholl, W. Rea, Y. Yang, and E.A. Stein, Synchronized delta oscillations correlate with the resting-state functional MRI signal, Proc Natl Acad Sci USA 104 (2007), 18265-18269.

T. Maddess, M.E. McCourt, B. Blakeslee, and R.B. Cunningham, Factors governing the adaptation of cells in area-17 of the cat visual cortex, Biol Cybern 59 (1988), 229-236.

A. Mechelli, C.J. Price, and K.J. Friston, Nonlinear coupling between evoked rCBF and BOLD signals: A simulation study of hemodynamic responses, Neuroimage 14 (2001), 862-872.
K.L. Miller, W.M. Luh, T.T. Liu, A. Martinez, T. Obata, E.C. Wong, L.R. Frank, and R.B. Buxton, Nonlinear temporal dynamics of the cerebral blood flow response, Hum Brain Mapp 13 (2001), 1-12.

M. Miller, P. Pasik, and T. Pasik, Extrageniculostriate vision in the monkey. VII, Contrast sensitivity functions. J Neurophysiol 43 (1980), 1510-1526.

S. Momjian, M. Seghier, M. Seeck, and C.M. Michel, Mapping of the neuronal networks of human cortical brain functions, Adv Tech Stand Neurosurg 28 (2003), 91-142.

J.R. Muller, A.B. Metha, J. Krauskopf, and P. Lennie, Rapid adaptation in visual cortex to the structure of images, Science 285 (1999), 14051408.

J.R. Muller, A.B. Metha, J. Krauskopf, and P. Lennie, Information conveyed by onset transients in responses of striate cortical neurons, J Neurosci 21 (2001), 6978-6990.

J. Niessing, B. Ebisch, K.E. Schmidt, M. Niessing, W. Singer, and R.A. Galuske, Hemodynamic signals correlate tightly with synchronized gamma oscillations, Science 309 (2005), 948-951.

T. Obata, T.T. Liu, K.L. Miller, W.M. Luh, E.C. Wong, L.R. Frank, and R.B. Buxton, Discrepancies between BOLD and flow dynamics in primary and supplementary motor areas: Application of the balloon model to the interpretation of BOLD transients, Neuroimage 21 (2004), 144-153.

S. Ogawa, D.W. Tank, R. Menon, J.M. Ellermann, S.G. Kim, H. Merkle, and K. Ugurbil, Intrinsic signal changes accompanying sensory stimulation: Functional brain mapping with magnetic resonance imaging, Proc Natl Acad Sci USA 89 (1992), 5951-5955.

W.W. Orrison, L.E. Davis, G.W. Sullivan, F.A. Mettler Jr., and E.R. Flynn, Anatomic localization of cerebral cortical function by magnetoencephalography combined with MR imaging and CT, AJ. NR Am. J Neuroradiol 11 (1990), 713-716.

D.G. Pelli, The VideoToolbox software for visual psychophysics: Transforming numbers into movies, Spat Vis 10 (1997), 437-442.

J. Pfeuffer, J.C. McCullough, P.F. Van de Moortele, K. Ugurbil, and X. Hu, Spatial dependence of the nonlinear BOLD response at short stimulus duration, Neuroimage 18 (2003), 990-1000.

C.J. Price and K.J. Friston, The temporal dynamics of reading: a PET study, Proc Biol Sci 264 (1997), 1785-1791.

M.D. Robson, J.L. Dorosz, and J.C. Gore, Measurements of the temporal fMRI response of the human auditory cortex to trains of tones, Neuroimage 7 (1998), 185-198.

E. Seidemann, A.B. Poirson, B.A. Wandell, and W.T. Newsome, Color signals in area MT of the macaque monkey, Neuron 24 (1999), 911-917.

R.G. Stockwell, L. Mansinha, and R.P. Lowe, Localization of the complex spectrum: The S-Transform, IEEE Trans Signal Process 44 (1996), 9981001.

A.L. Vazquez and D.C. Noll, Nonlinear aspects of the BOLD response in functional MRI, Neuroimage 7 (1998), 108-118. 